\title{
Uso de bentonita sódica como pretratamiento a la microfiltración tangencial de vino de mora de Castilla Rubus glaucus Benth
}

\section{(Use of sodium bentonite as a pretreatment to the tangential microfiltration of Castilla's blackberry wine Rubus glaucus Benth)}

\author{
Mariño Gabriel ${ }^{1}$, Coronel Manuel ${ }^{1}$, González Carlos $^{1}$, Elena Beltrán ${ }^{1}$
}

\begin{abstract}
Resumen:
Se estudió la influencia de técnicas de clarificación en vino de mora de Castilla Rubus glaucus Benth sobre su cromaticidad, turbidez y rendimientos del proceso. Inicialmente con la microfiltración tangencial (MFT) a tres diferentes presiones de trabajo (1.5, 2.0 y 2.5 bar) y complementariamente con el pretratamiento de la alimentación con bentonita sódica. La MFT a 1.5 bar obtuvo el mayor Factor de Retención Volumétrica (FRV) y el mejor rendimiento, estandarizando las condiciones de presión para la MFT con pretratamiento. En función del pH, en 9 gramos se estableció la cantidad óptima de floculante añadido a cada lote de vino, llevado a reposo a $6^{\circ} \mathrm{C}(6,12$ y 24 horas), obteniéndose $610 \pm 38 \mathrm{~g}$ para la mayor proporción de turbios en el lote de 6 horas de reposo, producto de una mayor captación inestable por parte del clarificante. La MFT de los pretratamientos indica mejoras representativas de hasta $40 \%$ en el rendimiento. Todas las muestras analizadas de vino microfiltrado con y sin pretratamiento, lograron una turbidez menor a 2 NTU, lo cual cumple con el criterio de calidad de "tinto brillante", y se concluye que la MFT por separado y con pretratamiento, mejoran la calidad del producto, pero pueden provocar deterioros en el color.
\end{abstract}

Palabras clave: microfiltración tangencial; vino; mora de Castilla Rubus glaucus Benth; bentonita.

\begin{abstract}
:
This research studied the influence of clarification techniques of Castilla's blackberry wine Rubus glaucus Benth on its chromaticity, turbidity and yields of the process. Initially, the tangential microfiltration (TMF) at three different working pressures $(1.5,2.0$ and $2.5 \mathrm{bar}$ ) and then the treatment of the feed with sodium bentonite prior the filtration. The TMF at 1.5 bar obtained the highest Volume Reduction Factor (VRF) and the best performance, standardizing the pressure conditions for the TMF with pretreatment. According the $\mathrm{pH}$, the optimum quantity of flocculant added to each batch of wine was established at 9 grams, carried to rest at $6^{\circ} \mathrm{C}(6,12$ and 24 hours), obtaining $610 \pm 38$ $\mathrm{g}$ for the highest proportion of turbid in the batch of 6 hours of rest, product of a greater unstable catch by the clarifier. The TMF of the pretreated batches indicated representative improvements in the yield, up to $40 \%$. All the analyzed microfiltered wine samples with and without pretreatment achieved a turbidity less than 2 NTU, accomplishing with the quality criterion of "bright red", concluding that the TMF separately and with pretreatment improve the quality of the product, but they can cause color deterioration.
\end{abstract}

Keywords: crossflow; tangential microfiltration; wine; Castilla's blackberry Rubus glaucus Benth; bentonite.

1 Universidad Tecnológica Equinoccial, Quito - Ecuador (\{mbga31291, manuel.coronel carlose.gonzalez, elena.beltran\} @ute.edu.ec). 


\section{Introducción}

La calidad, aceptación y preferencias de un vino, son atributos relacionados con estímulos percibidos por los sentidos del consumidor; son el color y la translucidez, las primeras características sometidas a valoración y simultáneamente adoptadas como indicadores del tipo, edad, procesos de elaboración y posibles defectos del producto (Coronel, 2010). Es indispensable la utilización de técnicas de clarificación como sedimentación espontánea, uso de aditivos, centrifugación o filtración, para reducir la cantidad de compuestos coloidales de alto peso molecular (taninos, pectinas y manoproteínas) conocidos como solidos insolubles en suspensión (s.i.s.), "turbios" o "enturbiamientos", responsables de causar una translucidez no deseada en el producto final (Arozarena, 2007)]. En etapas de reposo y a través de procedimientos inestables e insuficientes, se observa una tendencia de los vinos a clarificarse por la precipitación espontánea y natural de los s.i.s.; son necesarios prolongados períodos para que se adquiera la limpidez y estabilidad requerida por los estándares de calidad (Molina, 2000). Una clarificación "inducida", es la incorporación de sustancias floculantes o "clarificantes" que reducen la carga de s.i.s. (generalmente de carga positiva), debido a la atracción electroestática de los enturbiamientos con el aditivo (carga negativa), lo cual forma un complejo, capaz de sedimentarse en etapas de reposo y ser separado por medios físicos, siempre y cuando exista un diferencial entre las densidades de la partícula y del medio líquido. Su alta capacidad de estabilización y adsorción de compuestos que afectan a la limpidez, hacen del uso de bentonitas una alternativa adecuada para procesos de clarificación (Arozarena, 2007; Ribéreau-Gayon, Glories, Maujean, \& Dubourdieu, 2006).

La Microfiltración tangencial (MFT) es un proceso de separación, generado por una fuerza motriz o impulsora (presión, concentración, potencial eléctrico, etc.) sobre un medio filtrante poroso semipermeable conocido como "membrana", que favorece la transferencia o transporte de uno o más componentes y restringe el paso de otros a través de ella (permeabilidad selectiva), caracterizado por la dirección de flujo de sus corrientes. En función de la disposición de la membrana, el tránsito del volumen filtrado o de "permeado" $(\mathrm{Vp})$ es tangencial y para el volumen de "alimentación" ( $\mathrm{Va}$ ) sometido a filtración es paralelo, al igual que la corriente que no pudo superarla, conocida como "retenido" o concentrado (Vr) (Figura 1). La permeabilidad selectiva de la membrana está determinada por el tamaño de sus poros y para MFT se trabaja con diámetros que oscilan entre $0.1 \mathrm{a}$ $10 \mu \mathrm{m}$. Los principales parámetros de operación, son mediciones y factores concernientes al tipo de membrana, tamaño de poro (permeabilidad selectiva), presión transmembrana (PTM), temperatura, volumen de alimentación y de permeado (Benítez, 2012; Laverde, 2010; Raventós, 2005).

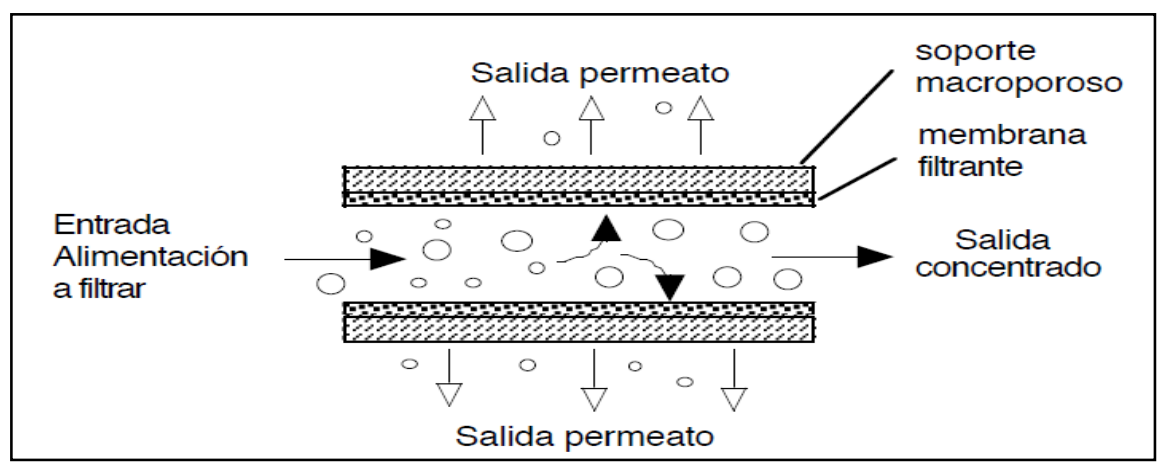

Figura 1. Esquema del principio de microfiltración tangencial 
El flujo transmembrana o flux de permeado $\left(J_{p}\right)$ es la expresión de capacidad del proceso, en función del volumen de permeado $\left(V_{p}\right)$ que atraviesa la membrana por unidad de área $\left(A_{m}\right)$ en transcurso del tiempo (dt) y limitada por la formación de un depósito irreversible de solutos sobre el medio filtrante, conocida como "colmatación" o ensuciamiento (Bacchin, Espinasse, \& Aimar, 2005). La presión transmembrana (PTM) es la fuerza motriz necesaria para el transporte de soluto a través del medio filtrante, proporcional al flujo e inversamente proporcional al tamaño del poro. Se define como el promedio de la presión de entrada $\left(\mathrm{P}_{\mathrm{e}}\right)$ y salida $\left(\mathrm{P}_{\mathrm{s}}\right)$ al módulo de filtración tangencial, menos la presión del flujo de permeado $\left(P_{p}\right)$ (Yacelga, 2007). El incremento de la PTM puede favorecer la colmatación irreversible de la membrana, lo cual provoca descensos pronunciados y no lineales del flux, es decir, el punto donde se superan las condiciones óptimas de filtración, conocidas como PTM y Flux críticos (Benítez, 2012; Laverde, 2010; Rodríguez, 2011; Yacelga, 2007). El factor de retención volumétrica (FRV) describe el efecto de concentración del volumen de Retenido $(\mathrm{Vr})$ o el rechazo del medio filtrante respecto a un soluto presente en la Alimentación (Va) (Montero, 2008; Yacelga, 2007). A su vez, como indicador de eficiencia industrial, el FRV nos permite calcular el rendimiento del proceso, entre el volumen de alimentación y de permeado (Montero, 2008).

El color de un vino es un indicador directo de calidad, químicamente ligado a los compuestos fenólicos presentes en las frutas, que permite evaluar las diferentes etapas de elaboración, estado de las materias primas, alteraciones, defectos, etc. (Olivero, 2006). La teoría del color es la unificación de métodos, medidas y escalas en condiciones estándar regidas por la CIE (Commission internationale de l'éclairage - "Comisión Internacional de la lluminación"), para la obtención de resultados cualitativos, cuantitativos y comparables, con todo lo relacionado al fenómeno físico de la percepción visual de los colores y sus propiedades de "cromaticidad" (Tono, Saturación y Luminosidad) (Maldonado, 2005; Coronel, 2010; Vilavella, 1997).

La Turbidez, es la medida que se utiliza para describir la limpidez de un vino, en función de la cantidad de s.i.s., presentes y expresada en forma de unidades nefelométricas de turbidez (NTU), generalmente obtenidas por comparación con una suspensión de sílice. Como referencia para estas mediciones, se han desarrollado parámetros de turbidez que vinculan la lectura de NTU con el aspecto (Tabla 1). Un vino tinto de calidad o "brillante" es considerado cuando su turbidez es menor a 2 NTU y "turbio" cuando esta supera los 8 NTU (Vilavella, 1997).

Tabla 1. Comparación de turbidez (NTU) con el aspecto del vino

\begin{tabular}{|l|c|}
\hline Aspecto del vino & Intervalo de turbidez (NTU) \\
\hline Brillante & $0.4-1.5$ \\
\hline Claro & $1.5-2.5$ \\
\hline Velado & $2.5-5.0$ \\
\hline Opalescente & $5.0-15$ \\
\hline Turbio & $>15$ \\
\hline \multicolumn{2}{|c|}{ (Vilavella, 1997) } \\
\hline
\end{tabular}

\section{Metodología}

Para su desarrollo y análisis, el presente estudio fue separado en dos partes fundamentales. La primera para determinar a qué presión de trabajo se alcanzó el mayor rendimiento o FRV, de 3 microfiltraciones de vino de mora de Castilla Rubus glaucus Benth realizadas a 1.5, 2.0 y 2.5 bar, respectivamente, con la finalidad de fijarla como presión de operación óptima para la segunda fase, donde se realizó el tratamiento de la alimentación con bentonita sódica previo a la MFT a diferentes tiempos de reposo (6, 12, y 24 horas). 


\section{Elaboración de Vino de Mora de Castilla}

Se elaboró vino de mora de Castilla Rubus glaucus Benth en un biorreactor de escala piloto, a partir de materia prima previamente caracterizada. En la Figura 2, se presentan las diferentes etapas del proceso.

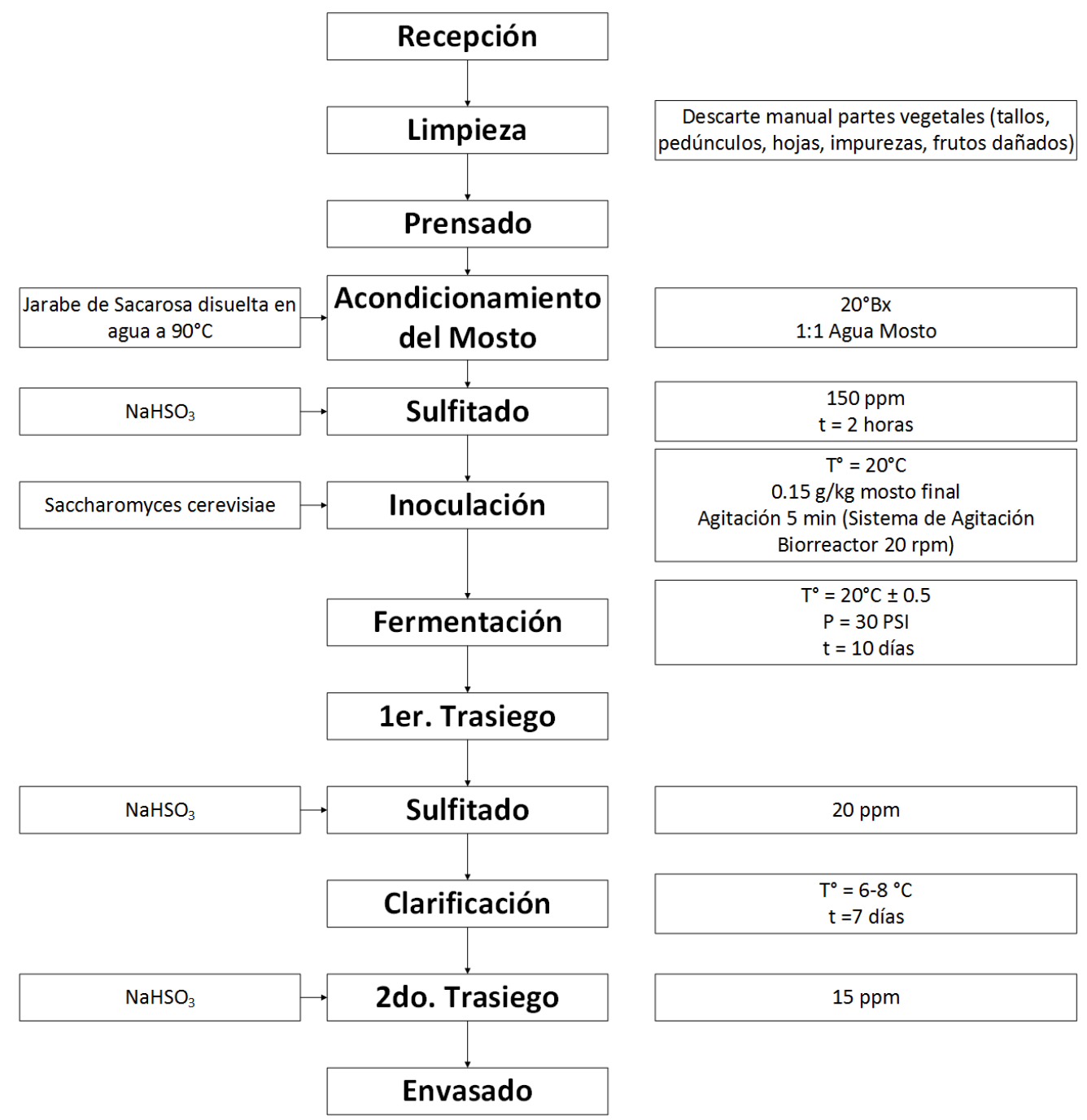

Figura 2. Proceso de elaboración de vino de mora de Castilla

(Clarke, 2001)

\section{Microfiltración Tangencial}

En esta fase se utilizó un equipo de MFT de escala piloto (Figura 3), compuesto de un módulo de filtración (E-5) que posee en su interior a la membrana de cerámica marca Pal (USA) (108 cm de largo, superficie de filtración de $0.36 \mathrm{~m}^{2}, 19$ canales, diámetro de poro de $0.45 \mu \mathrm{m}$ de diámetro, resistente a 10 bar de presión y $95^{\circ} \mathrm{C}$ de temperatura máxima) recubierta por una carcasa de acero inoxidable AISI 310 y un sistema de refrigeración por chaqueta provisto de agua a temperatura ambiente $\left(T \approx 18^{\circ} \mathrm{C}\right)(\mathrm{E}-6)$, para mantener las propiedades físico-químicas de la alimentación, cuando su temperatura aumenta por la fricción generada con la membrana. Además, el equipo posee una bomba centrífuga (E-4), bomba de diafragma (capacidad de 8 bar) (E-3), unidad móvil de aire comprimido (E-1), sistema de control de presión $(\mathrm{V}-1)$, manómetros $(\mathrm{I}-1 ; \mathrm{I}-2)$, termómetros(I-3), tuberías (AISI 310) y válvulas sanitarias (V-1; V-2; V-3). La capacidad volumétrica del equipo es de $6 \mathrm{~L}$ y de la membrana $1.5 \mathrm{~L}$. 


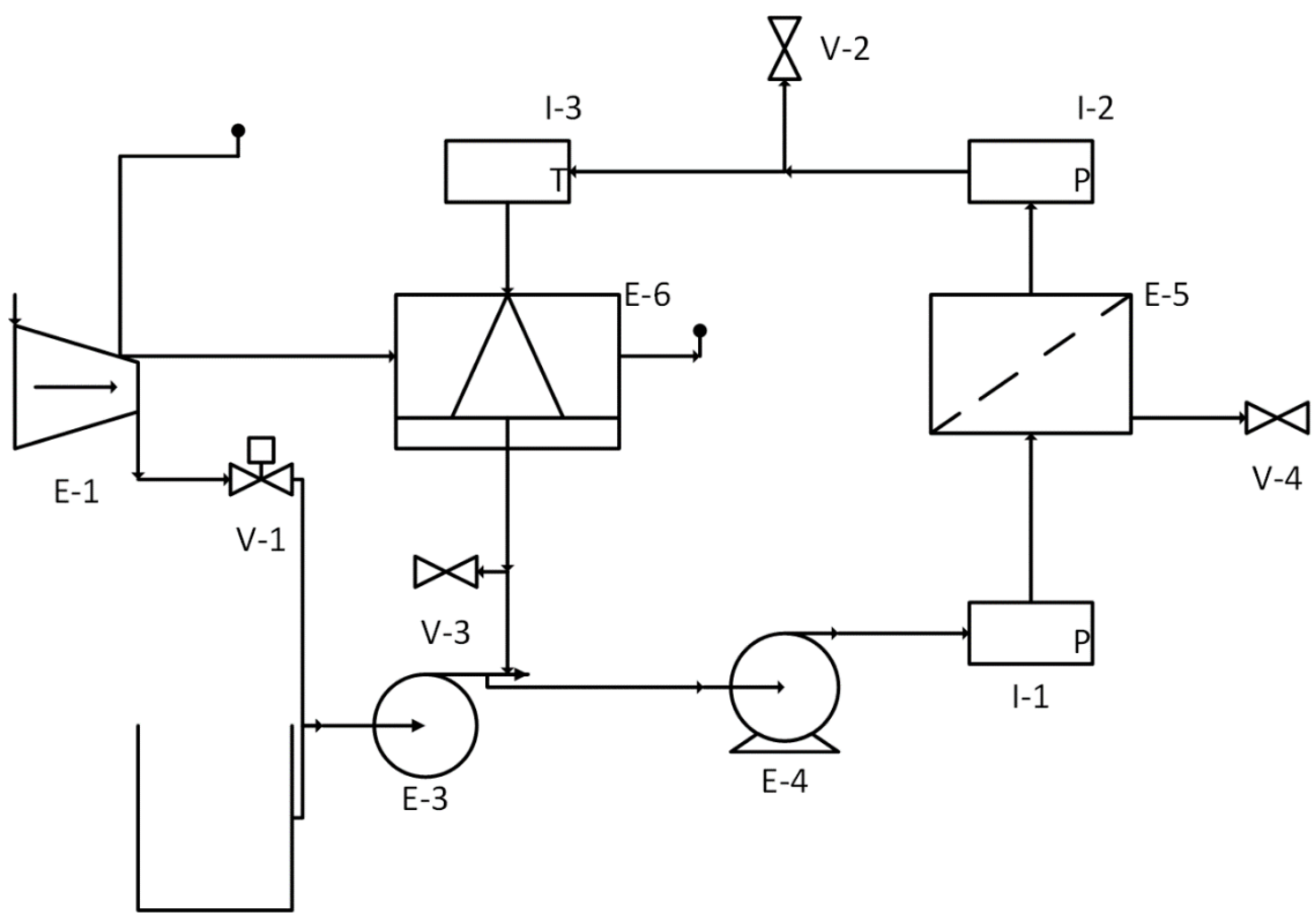

$\mathrm{E}-2$

Figura 3.Esquema del equipo de microfiltración tangencial

Se siguieron los protocolos de uso y limpieza, para recuperar la permeabilidad inicial de la membrana en cada sesión; la primera parte del estudio realizó por duplicado la MFT de tres lotes de vino de mora de Castilla de 15 litros cada uno, a tres niveles de presión de trabajo (1.5, 2.0 y 2.5 bar) para determinar su respectivo FRV (5). Por comparación de dichas mediciones, la presión de operación correspondiente al valor mayor de FRV, fue establecida como la presión única de trabajo para la segunda etapa de la investigación.

Por separado y con su respectiva réplica, la segunda parte del estudio utilizó cuatro lotes de vino de mora de Castilla de $10 \mathrm{~L} \mathrm{c/u}$, tres de ellos con una cantidad específica de bentonita añadida como tratamiento en función de su pH (Tabla 2) y el lote restante como testigo sin tratamiento. Para mejorar su disponibilidad y difusión, la bentonita previamente requirió ser hidratada bajo agitación de 10 minutos, a una relación de 1:7.5 partes de bentonita y agua, hasta la obtención de un gel estable. Cada lote fue trasladado a refrigeración a $6^{\circ} \mathrm{C}$ y sometido a reposo de 6,12 y 24 horas, respectivamente. Después del reposo de cada contenedor, se procedió a trasegar el vino para separar los turbios decantados en el fondo del recipiente por acción del floculante y como paso final su microfiltración.

Tabla 2.Cantidad de Bentonita Sódica en función del pH

\begin{tabular}{|l|c|}
\hline Cantidad & $\mathbf{p H}$ \\
\hline $10 \mathrm{~g} / \mathrm{hL}$ & 2,3 \\
\hline $50 \mathrm{~g} / \mathrm{hL}$ & 3,0 \\
\hline $200 \mathrm{~g} / \mathrm{hL}$ & 3,6 \\
\hline \multicolumn{2}{|c|}{ (Molina, 2000) } \\
\hline
\end{tabular}

En cada uno de los procedimientos de MFT de la primera y segunda parte, se midieron los flujos y volúmenes de permeado, retenido y de alimentación (1), tiempo de 
operación, presiones de entrada y salida del módulo de microfiltración, para respectivamente calcular Flujo Transmembrana $J_{p}$ (2), PTM (3), FRV (4) y el rendimiento de la operación (5) de cada experimentación.

$$
\begin{aligned}
& V_{a}=V_{r}+V_{p} \\
& \mathrm{~J}_{\mathrm{p}}=\frac{1}{\mathrm{Am}} \frac{\mathrm{V}_{p}}{\mathrm{dt}} \\
& \mathrm{PTM}=\frac{\mathrm{P}_{\mathrm{e}}+\mathrm{P}_{\mathrm{s}}}{2}-\mathrm{P}_{\mathrm{p}} \\
& \mathrm{FRV}=\frac{\mathrm{V}_{\mathrm{a}}}{\mathrm{V}_{\mathrm{r}}}=\frac{\mathrm{V}_{\mathrm{r}}+\mathrm{V}_{\mathrm{p}}}{\mathrm{V}_{\mathrm{r}}}=1+\frac{\mathrm{V}_{\mathrm{p}}}{\mathrm{V}_{\mathrm{r}}} \\
& \text { Rendimiento }=\frac{\mathrm{Vp}}{\mathrm{Va}}=1-\frac{1}{\mathrm{FRV}}
\end{aligned}
$$

\section{Medición del color}

\section{Espectrofotometría}

Con un espectrofotómetro marca Thermo Scientific - Evolution 60S UV-Visible en modo de barrido o escaneo, se realizaron lecturas de absorbancias en un rango espectral de 380 a $780 \mathrm{~nm}$, para cada muestra y por triplicado. Las mediciones obtenidas fueron analizadas mediante el modelo matemático de Glories (7) (8) (9) (10) (11), para determinar los parámetros cromáticos en vinos tintos de Intensidad Colorante (IC), Tono (T) y porcentajes de color amarillo, azul y rojo. (Ribéreau-Gayon et al., 2006)

$$
\begin{aligned}
& \mathrm{IC}=\mathrm{A}_{420}+\mathrm{A}_{520}+\mathrm{A}_{620} \\
& \mathrm{~T}=\mathrm{A}_{420} / \mathrm{A}_{520} \\
& \% \text { Amarillo }=\left(\mathrm{A}_{420} / \mathrm{IC}\right) * 100 \\
& \% \text { Rojo }=\left(\mathrm{A}_{520} / \mathrm{IC}\right) * 100 \\
& \% \text { Azul }=\left(\mathrm{A}_{620} / \mathrm{IC}\right) * 100
\end{aligned}
$$

\section{Turbidez}

Para la toma de las mediciones se empleó un Turbidímetro o Nefelómetro marca Hach 2100P (0 - 1000 NTU), capaz de determinar la intensidad de la luz dispersada a 90 grados, cuando un rayo de luz atraviesa una muestra de agua.

\section{Diseño Experimental}

El estudio de microfiltración de vino de mora de Castilla a diferentes presiones de trabajo con su respectiva réplica, utilizó un diseño unifactorial completamente al azar para comparar el proceso, empleando como variable dependiente al FRV e independientes a los tres niveles de presión de trabajo (1.5, 2.0 y 2.5 bar). De igual forma, la segunda parte del estudio y su réplica, utilizaron un diseño unifactorial completamente al azar para contrastar un pretratamiento de la alimentación a la MFT con bentonita sódica; se adoptaron como variables dependientes al FRV e independientes a los pretratamientos con floculante (PT): Sin Bentonita "SB" (testigo), Con Bentonita y reposo 6 horas "CB-6h", Con Bentonita y reposo 12 horas "CB-12h", Con Bentonita y reposo 24 horas "CB-24h". Los resultados y mediciones obtenidas de espectrofotometría y turbidez en todas las muestras, 
fueron examinadas con un análisis de varianza simple "ANOVA", a través de la prueba de Tukey con un nivel de confianza del $95 \%$, a partir de 5 determinaciones de cada muestra.

\section{Resultados y Discusión}

\section{MFT de Vino de mora de Castilla a 3 niveles de presión.}

Contrariamente a lo observado en la Tabla 3, donde el flujo transmembrana mayor se produce a la presión de 2.0 bar, su FRV es menor en relación con el más alto (1.5 bar con 1.71 de FRV). El proceso con mayor FRV, indicó la mayor proporción de retención de la membrana sobre los s.i.s de la alimentación (superiores a $0.45 \mu \mathrm{m}$ ) y simultáneamente permitió determinar la presión de operación única para la segunda etapa del estudio (1.5 bar), a partir de su respectiva dependiente. Existe una relación inversamente proporcional entre el FRV y el flujo transmembrana, producto de la permeabilidad selectiva y sobre todo por el incremento de la presión transmembrana por encima del punto crítico que aumenta la velocidad de crecimiento de la torta de filtración y tapona la membrana, perjudicando al flux (Cabrera \& Perlera, 2009; Chacón, 2005; Mendoza, 2010). Es necesario indicar que el volumen inicial de cada lote era 15 litros, pero solo se tomó como volumen real de alimentación a aquel que ingresó en el equipo hasta que el flujo transmembrana se estabilizó (5 mediciones iguales), es decir, cuando la membrana se colmató, cuando se obtuvieron $12 \pm 0.245,10.5 \pm 0.393$ y $9.7 \pm 0.154$ litros para $1.5,2.0$ y 2.5 bar de presión de trabajo, respectivamente.

Tabla 3. Parámetros de MFT de Vino de Mora de Castilla a 3 niveles de presión

\begin{tabular}{|c|c|c|c|c|c|}
\hline Presión de trabajo (bar) & $\mathbf{( P T M ) ~ ( b a r ) ~}$ & $\begin{array}{c}\mathbf{J P}_{\text {inicial }} \\
\left(\mathbf{I} / \mathbf{h}^{\star} \mathbf{m}^{\mathbf{2}}\right)\end{array}$ & $\mathbf{J P}_{\text {final }}\left(\mathbf{I} / \mathbf{h}^{\star} \mathbf{m}^{\mathbf{2}}\right)$ & FRV & Rendimiento \\
\hline $\mathbf{1 . 5 0}$ & $1.41 \pm 0.08$ & $39.44 \pm 0.35$ & $5.14 \pm 0.09$ & $1.71 \pm 0.08$ & $41.67 \% \pm 0.02$ \\
\hline $\mathbf{2 . 0 0}$ & $2.00 \pm 0.15$ & $58.68 \pm 0.56$ & $10.5 \pm 0.55$ & $1.62 \pm 0.03$ & $38.1 \% \pm 0.14$ \\
\hline $\mathbf{2 . 5 0}$ & $2.33 \pm 0.08$ & $45.58 \pm 0.45$ & $9.26 \pm 0.40$ & $1.45 \pm 0.07$ & $30.93 \% \pm 0.13$ \\
\hline
\end{tabular}

El comportamiento característico de una curva de Microfiltración para fluidos biológicos como el vino, es descrito por un descenso pronunciado y progresivo del flujo transmembrana, hasta alcanzar la estabilidad en un intervalo de tiempo (Mendoza, 2010), generado por los efectos de la polarización de los solutos y colmatación de la membrana. En la Figura 4, se observan comportamientos semejantes entre las microfiltraciones de cada presión, diferenciándose por los flux iniciales, pero con asíntotas similares al momento del ensuciamiento. Las variaciones de presión en MFT producen cambios en el FRV y flujos permeados, pero no significativamente en el grado de colmatación, cuando referenciamos el mismo tipo de alimentación (Mendoza, 2010).

\section{Pretratamiento con Bentonita Sódica}

La cantidad de bentonita aplicada en función del pH del vino (3.21) (Figura 5), se determinó en $9 \mathrm{~g}$ de bentonita por cada 10 litros (volumen de cada lote), a partir de la interpolación de los datos de la Tabla 2.

Por acción del floculante y separados por un proceso de trasiego, se obtuvieron 0.610 $\pm 0.038 \mathrm{~kg}, 0.491 \pm 0.013 \mathrm{~kg}$ y $0.307 \pm 0.064 \mathrm{~kg}$ de s.i.s de los tratamientos de 6,12 y 24 horas de reposo, respectivamente, observándose un descenso marcado del 20 y $50 \%$ en relación con el primero. Las bentonitas para clarificación de vinos remueven los compuestos de acuerdo con su punto isoeléctrico (pl) sin afectar las características organolépticas del producto, formando enlaces electroestáticos estables entre las sustancias que tengan el pl cercano o similar al de la bentonita $(8.5-10)$, pero perdiendo firmeza por el transcurso del tiempo o por cambios en el diferencial entre los puntos isoeléctricos (Olivero, 2006). Las proteínas son elementos presentes en el vino que 
producen mayor formación de enturbiamientos, potencialmente removidas con la adición de bentonita, porque poseen un pl entre 5.8 a 8 y forman enlaces firmes, a diferencia de los taninos y antocianinas (compuestos responsables del color), que crean enlaces menos estables, fácilmente descompuestos en sus elementos originales.

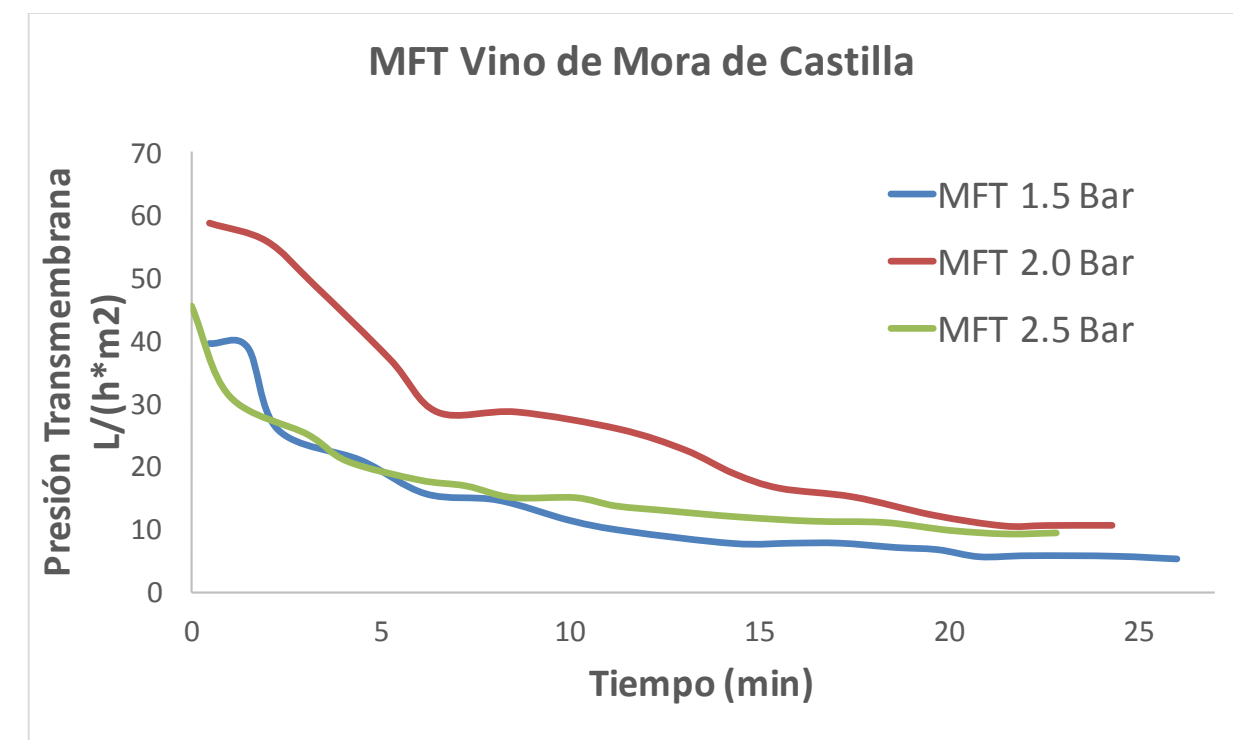

Figura 4. MFT de Vino de Mora de Castilla a 3 niveles de presión

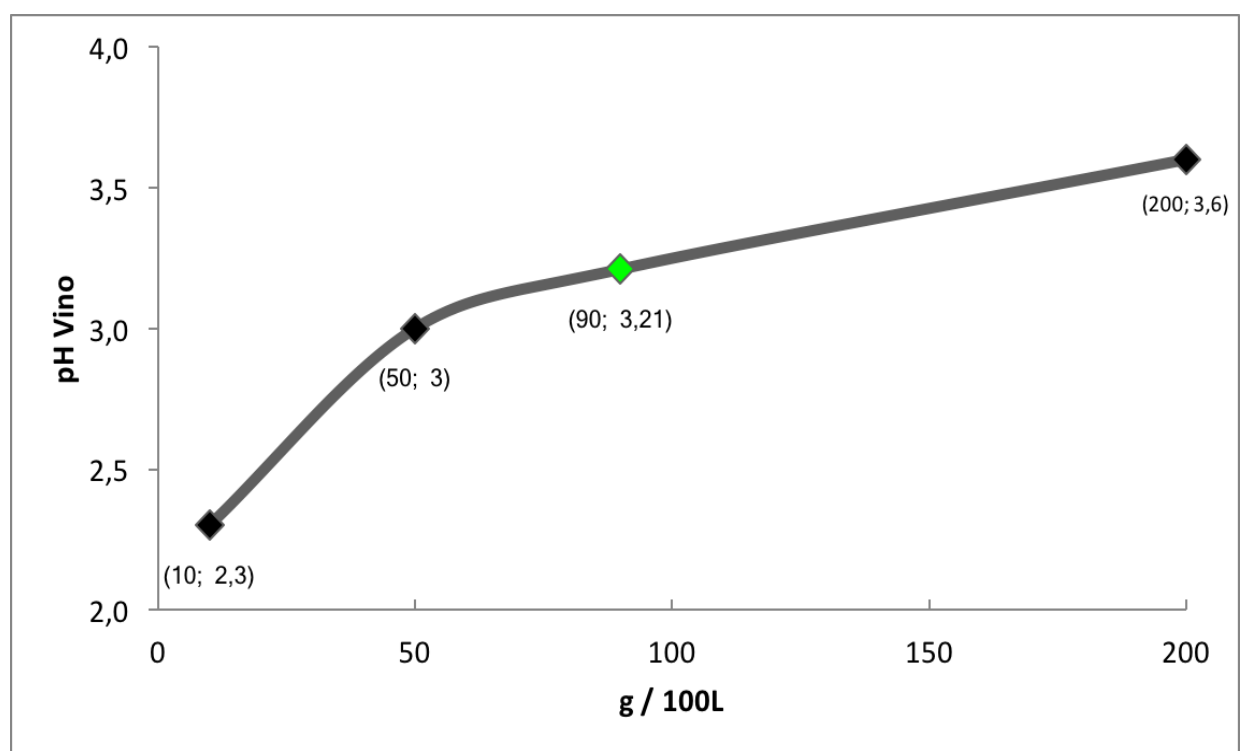

Figura 5.Cantidad de Bentonita en función del pH

\section{Microfiltración Tangencial de vino de Castilla con pretratamiento de bentonita sódica}

A partir de la MFT de $9.00 \pm 0.11 ; 9.00 \pm 0.05 ; 9.00 \pm 0.19$ y $9.50 \pm 0.20$ litros de alimentación para "SB", "CB-6h", "CB-12h", "CB-24h", correspondientemente, se obtuvieron los flux iniciales en los diferentes procedimientos y se concluyó que la bentonita como pretratamiento influye y mejora el proceso, en comparación con la MFT sin pretratamiento ( Tabla 4), lo cual provoca aumentos generalizados en el FRV, porcentajes de rendimiento y flujo transmembrana. La remoción de s.i.s., después del reposo de los lotes de vino con bentonita, aumentó el rendimiento de la MFT de hasta un $11.77 \%$ y $18.84 \%$ del FRV en el pretratamiento "CB-6h", originado por una mayor agrupación de varios compuestos en forma inestable que fueron removidos en el trasiego, sin embargo, 
se pudo observar una disminución cuando el tiempo aumenta (“CB-12h" y "CB-24h"), producida una floculación parcializada con las proteínas (estabilización proteica). Las partículas de bentonita hidratada poseen una red electronegativa, capaz de mantener enlaces firmes con proteínas (carga positiva) y en menor grado por su mayor diferencial en el potencial con otros compuestos (polifenoles, antocianinas, etc.), pero ambos afectados por el transcurso del tiempo (Cabrera et al., 2009).

Tabla 4.Parámetros de MFT de Vino de Mora con pretratamiento de bentonita sódica

\begin{tabular}{|l|c|c|c|c|c|c|}
\hline \multicolumn{1}{|c|}{ PT } & $\begin{array}{c}\text { Presión de } \\
\text { trabajo (bar) }\end{array}$ & $\mathbf{P T M}(\mathbf{b a r})$ & $\begin{array}{c}\mathbf{J P}_{\text {inicial }} \\
\left(\mathbf{I} / \mathbf{h}^{\star} \mathbf{m}^{2}\right)\end{array}$ & $\begin{array}{c}\mathbf{J P}_{\text {final }} \\
\left(\mathbf{I} / \mathbf{h}^{\star} \mathbf{m}^{2}\right)\end{array}$ & $\mathbf{F R V}$ & Rendimiento \\
\hline SB & 1.50 & $1.41 \pm 0.01$ & $32.1 \pm 0.09$ & $6.35 \pm 0.9$ & $1.38 \pm 0.01$ & $27.78 \% \pm 0.31$ \\
\hline CB-6h & 1.50 & $1.44 \pm 0.10$ & $61.3 \pm 0.06$ & $11.13 \pm 0.73$ & $1.64 \pm 0.06$ & $38.89 \% \pm 0.32$ \\
\hline CB-12h & 1.50 & $1.38 \pm 0.12$ & $67.5 \pm 0.79$ & $12.81 \pm 0.59$ & $1.5 \pm 0.08$ & $33.33 \% \pm 0.23$ \\
\hline CB-24h & 1.50 & $1.45 \pm 0.17$ & $102.1 \pm 0.68$ & $20.69 \pm 0.71$ & $1.45 \pm 0.05$ & $31.05 \% \pm 0.15$ \\
\hline
\end{tabular}

Para el caso de los vinos, donde la filtración tangencial por membranas no es totalmente efectiva sobre algunas partículas inestables capaces de originar nuevos enturbiamientos, se genera la necesidad de procesos previos de clarificación mediante la adición de sustancias floculantes y posteriormente provocar aumentos en el rendimiento, disminuir costos de producción, reducir la colmatación, mejorar estabilidad y calidad sensorial del producto (Molina, 2000). La Figura 6, describe el aumento significativo de los flujos transmembrana mínimos y máximos de las MFT con pretratamiento en relación con el testigo, observándose un incremento de los flux iniciales desde 91 a $318 \%$ y también para la zona de colmatación entre un 75 a $326 \%$. Los complejos formados entre bentonita, proteína y otros compuestos, fueron removidos en el trasiego previo a la filtración, lo cual redujo la cantidad de s.i.s., de la alimentación y su consecuente aumento en el rendimiento del proceso.

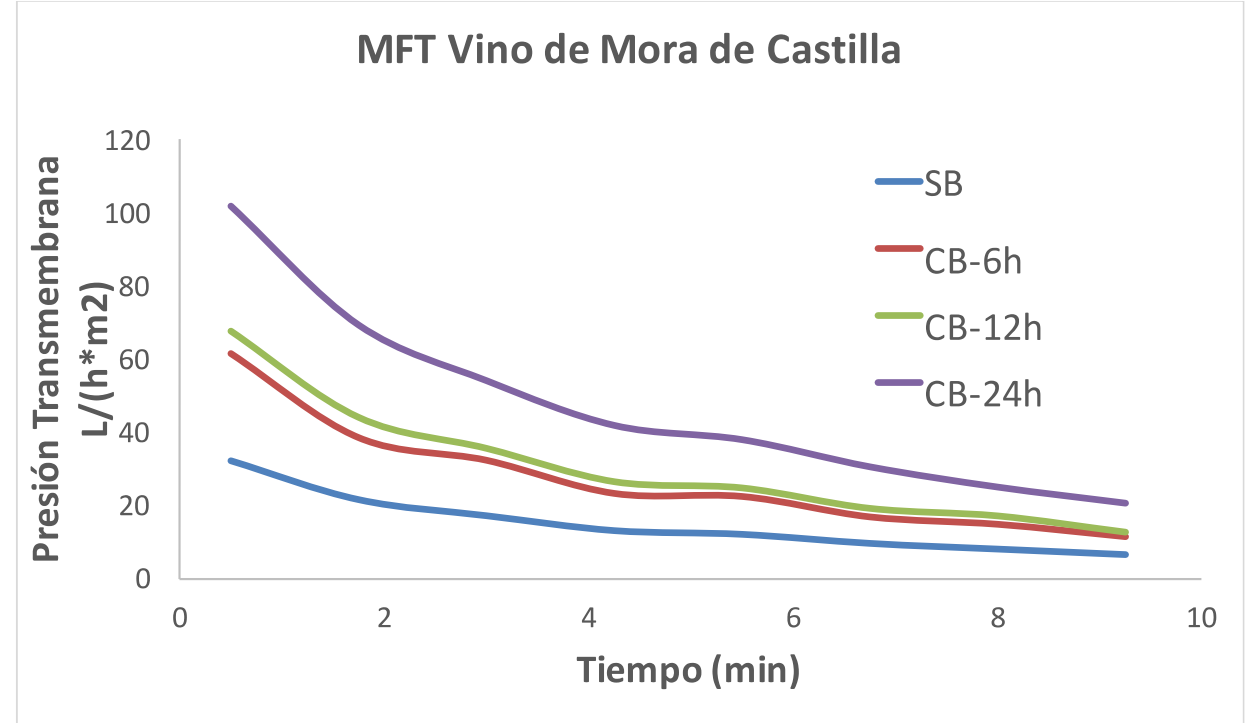

Figura 6. MFT de Vino de Mora con pretratamiento y muestra testigo

\section{Análisis de color}

\section{Método Glories}

En la Figura 7 se representa el análisis de color para muestras microfiltradas a 3 diferentes presiones de trabajo, por medio de espectrofotometría con sus respectivos promedios de absorbancias. Se comprobó que las cuatro muestras tienen su pico máximo entre 515 y $520 \mathrm{~nm}$, lo cual describe una indirecta y cualitativa composición fenólica con 
presencia de antocianinas, responsables del color característico de los vinos tintos. Por otra parte visualmente imperceptible, se determinó un decrecimiento representativo de la absorbancia por efecto de la colmatación de la membrana, cuando la presión de operación supera las condiciones críticas de flujo transmembrana, lo cual evidencia una mayor retención de compuestos de color. La retención de antocianinas en MFT en ciertas condiciones puede ser nula, pero puede ser afectada por una colmatación irreversible o porque fueron atrapadas por algún tipo de s.i.s., que no sobrepasarán el medio filtrante (Peri, Riva, \& Decio, 1988).

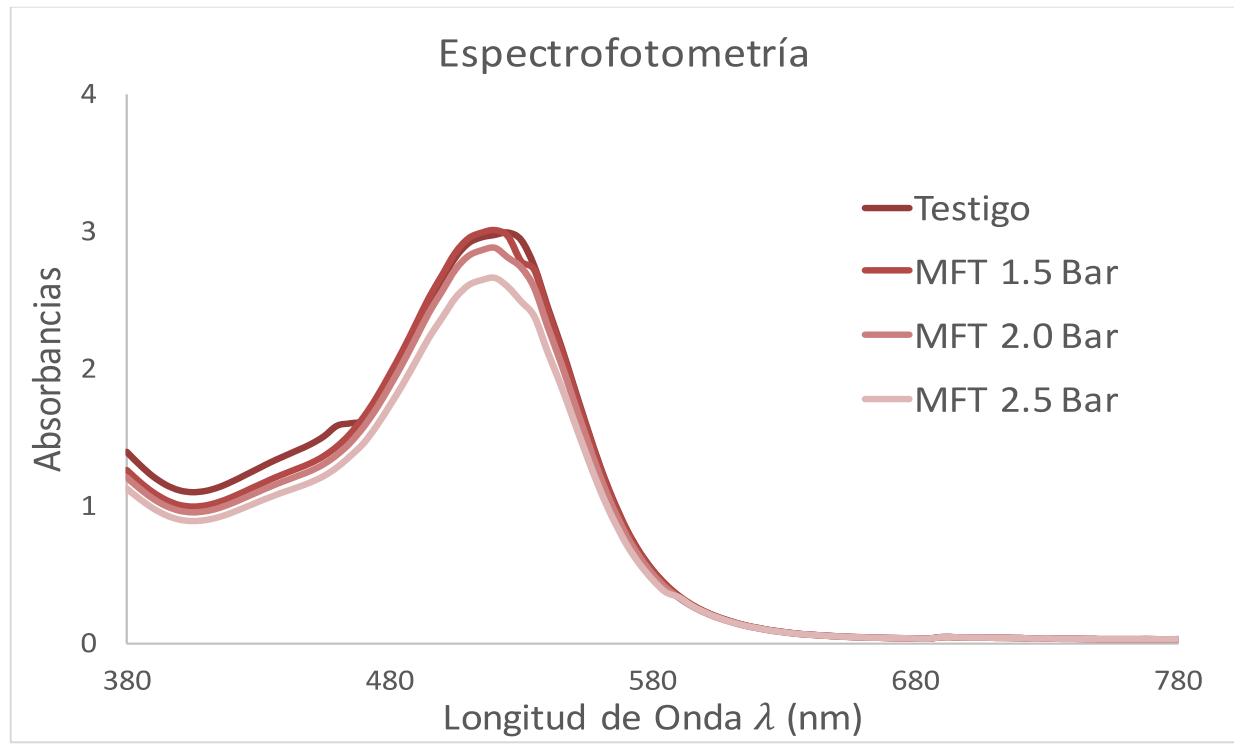

Figura 7. Espectrofotometría de MFT a diferentes niveles de PTM

El método Glories para análisis de color en muestras de vino microfiltrado a tres diferentes niveles de presión de trabajo (Tabla 5), presenta las características cromáticas de Intensidad Colorante (7) y Tono (8), con sus respectivos porcentajes de amarillo (9), rojo (10) y azul (11). La detención de compuestos responsables de color en la MFT no afectó al tono de las muestras, pero ejerció una influencia significativa sobre las absorbancias a 420 y $520 \mathrm{~nm}$, lo cual estableció grupos heterogéneos sobre la IC de las muestras, es decir, la filtración altera la saturación del color, principalmente al porcentaje de azul. Una desventaja de la microfiltración de vinos es la colmatación irreversible, reflejada en la retención de proteínas y fenoles como antocianinas, capaces de taponar la membrana y originar reducciones en la tasa de flux y deterioros en el color (Mendoza, 2010; Ribéreau-Gayon, et al., 2006).

En la espectrofotometría de las muestras microfiltradas con pretratamiento de bentonita (Figura 8), se observó que el valor máximo de absorbancia de cada muestra permanece entre 515 y $520 \mathrm{~nm}$, sin desplazamientos provocados por efecto de la bentonita o por la MFT, no obstante, se pudo determinar que el pretratamiento afectó el nivel de absorbancia en dichas longitudes de onda, y presentó a "SB" (b) y "CB-6h" (a) como grupos heterogéneos y a "CB-12h" (c) con "CB-24h" (c) como homogéneos, lo cual describió una desagregación considerable de los compuestos responsables de color procedentes de los complejos formados con el floculante, cuando se incrementa el tiempo de reposo. Indirectamente los complejos formados de proteína y bentonita pueden unirse con antocianinas y generar pérdidas de color (Gómez, Gil, López, M. De La Hera, \& Martínez, 2000). 
Tabla 5. Método Glories para la MFT a 3 niveles de presión

\begin{tabular}{|c|c|c|c|c|}
\hline Presión & Testigo & $1.5 \mathrm{bar}$ & $2.0 \mathrm{bar}$ & 2.5 bar \\
\hline$\lambda=420 \mathrm{~nm}$ & $1.18 \pm 0.00^{d}$ & $1.06 \pm 0.02^{c}$ & $1.02 \pm 0.00^{b}$ & $0.95 \pm 0.01^{a}$ \\
\hline$\lambda=520 \mathrm{~nm}$ & $2.97 \pm 0.00^{c}$ & $3.00 \pm 0.05^{c}$ & $2.87 \pm 0.01^{b}$ & $2.66 \pm 0.02^{a}$ \\
\hline$\lambda=620 \mathrm{~nm}$ & $0.11 \pm 0.00^{b}$ & $0.11 \pm 0.00^{b}$ & $0.11 \pm 0.00^{a}$ & $0.11 \pm 0.00 \mathrm{ab}$ \\
\hline IC & $4.26 \pm 0.00^{c}$ & $4.17 \pm 0.07^{c}$ & $3.99 \pm 0.02^{b}$ & $3.73 \pm 0.03^{a}$ \\
\hline Tono (T) & $0.40 \pm 0.00^{a}$ & $0.35 \pm 0.00^{a}$ & $0.35 \pm 0.00^{a}$ & $0.36 \pm 0.00^{a}$ \\
\hline \% Amarillo & $27.61 \pm 0.00^{a}$ & $25.45 \pm 0.00^{a}$ & $25.45 \pm 0.00^{a}$ & $25.57 \pm 0.00^{a}$ \\
\hline \% Rojo & $69.71 \pm 0.00^{a}$ & $71.82 \pm 0.00^{a}$ & $71.83 \pm 0.00^{a}$ & $71.45 \pm 0.00^{a}$ \\
\hline$\%$ Azul & $2.68 \pm 0.00^{a}$ & $2.73 \pm 0.00^{c}$ & $2.73 \pm 0.00^{b}$ & $2.98 \pm 0.00^{d}$ \\
\hline
\end{tabular}

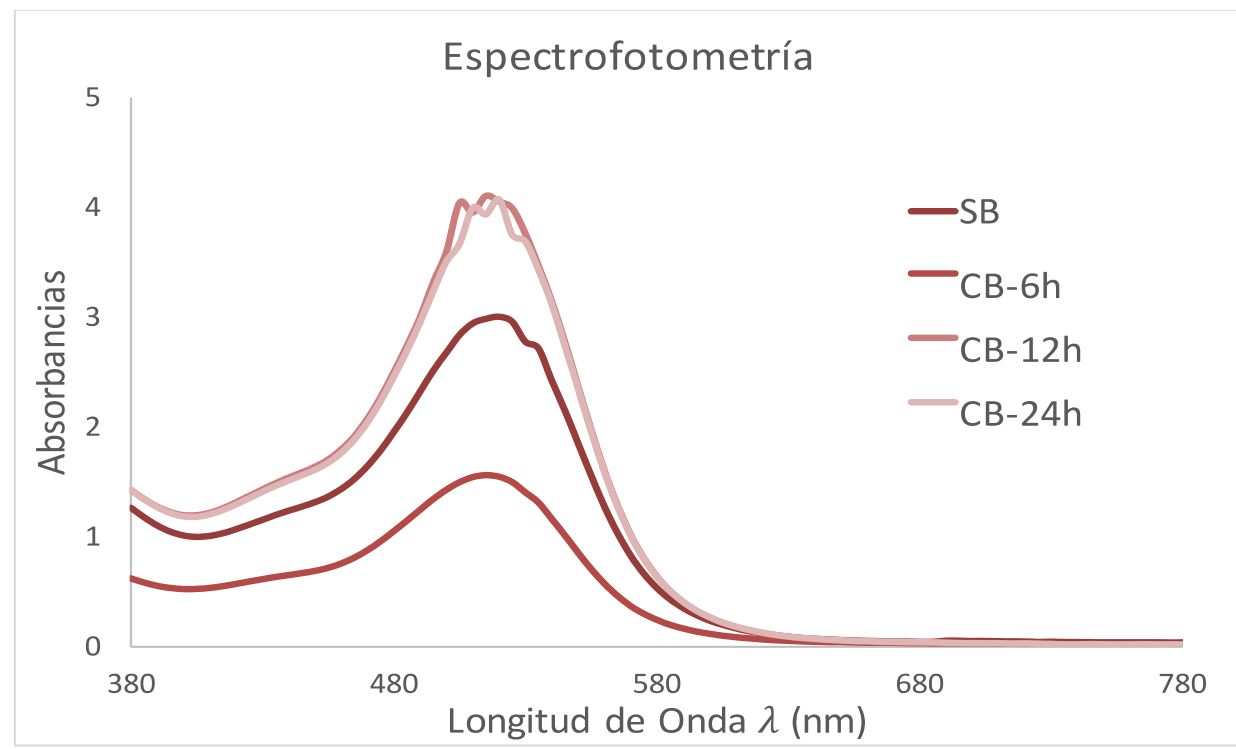

Figura 8. Espectrofotometría de Pretratamientos

En la Tabla 6, se exhiben los parámetros cromáticos calculados a través del método Glories, para muestras microfiltradas con pretratamiento de bentonita. El cambio considerable pero imperceptible a la vista de las absorbancias a 420 y $520 \mathrm{~nm}$, provocaron variaciones sobre los porcentajes de azul, rojo y amarillo, con un consecuente cambio del Tono para las muestras de 12 y 24 horas de reposo. La Intensidad Colorante presentó mayores diferencias significativas en comparación con la muestra testigo, alcanzando una mayor reducción para "CB-6h" de 48\%, dependiente de una gran cantidad de complejos inestables formados entre los s.i.s., con la bentonita y a su vez con los compuestos responsables de color, pero, cuando sus enlaces pierden fuerza por paso del tiempo, únicamente se observará una interacción con los s.i.s., que serán removidos mediante procesos físicos después del reposo y permitirán que la absorbancia se incremente en la muestra, debido a que el paso de la luz en su presencia es obstaculizado en mayor proporción. La adición de clarificantes en la elaboración de vinos, disminuyen la intensidad colorante sin generar cambios significativos en el tono del producto (Gómez et al., 2000).

\section{Turbidez}

El vino de mora de Castilla clasificado como tinto (rojo rubí), utiliza la categoría de "tinto brillante" (turbidez $\leq 2$ NTU), como criterio de calidad comercial (Tabla 2) (Vilavella, 1997)]. En las muestras microfiltradas se observó una reducción progresiva y significativa 
de la turbidez de hasta un $98 \%$ ( Tabla 7 ) y se alcanzaron los parámetros de calidad para todas ellas, pero con una heterogeneidad individual, producto de una mayor retención de solutos en la MFT a presiones que sobrepasan el flujo transmembrana crítico. La MFT permite obtener productos de alta estabilidad microbiológica, organoléptica y de limpidez (inferior a 1 NTU).

Tabla 6.Método Glories para la MFT de los diferentes pretratamientos

\begin{tabular}{|l|l|l|l|l|}
\hline PT & SB & CB-6h & CB-12h & CB-24h \\
\hline $\boldsymbol{A}=\mathbf{4 2 0} \mathbf{~ n m}$ & $1.06 \pm 0.017^{\mathrm{b}}$ & $0.56 \pm 0.009^{\mathrm{a}}$ & $1.31 \pm 0.006^{\mathrm{c}}$ & $1.28 \pm 0.005^{\mathrm{c}}$ \\
\hline $\boldsymbol{A}=\mathbf{5 2 0} \mathbf{~ n m}$ & $2.99 \pm 0.036^{\mathrm{b}}$ & $1.53 \pm 0.014^{\mathrm{a}}$ & $4.05 \pm 0.147^{\mathrm{c}}$ & $4.05 \pm 0.194^{\mathrm{c}}$ \\
\hline $\boldsymbol{\lambda}=\mathbf{6 2 0} \mathbf{~ n m}$ & $0.11 \pm 0.007^{\mathrm{b}}$ & $0.06 \pm 0.007^{\mathrm{a}}$ & $0.12 \pm 0.004^{\mathrm{b}}$ & $0.12 \pm 0.007^{\mathrm{b}}$ \\
\hline $\mathbf{I C}$ & $4.17 \pm 0.058^{\mathrm{b}}$ & $2.16 \pm 0.029^{\mathrm{a}}$ & $5.48 \pm 0.151^{\mathrm{c}}$ & $5.46 \pm 0.187^{\mathrm{c}}$ \\
\hline Tono (T) & $0.35 \pm 0.003^{\mathrm{b}}$ & $0.36 \pm 0.003^{\mathrm{b}}$ & $0.32 \pm 0.012^{\mathrm{a}}$ & $0.31 \pm 0.016^{\mathrm{a}}$ \\
\hline \% Amarillo $^{2}$ & $25.4 \pm 0.135^{\mathrm{b}}$ & $26.1 \pm 0.100^{\mathrm{b}}$ & $23.9 \pm 0.673^{\mathrm{a}}$ & $23.5 \pm 0.886^{\mathrm{a}}$ \\
\hline \% Rojo & $71.8 \pm 0.260^{\mathrm{a}}$ & $70.7 \pm 0.388^{\mathrm{a}}$ & $73.8 \pm 0.680^{\mathrm{b}}$ & $74.1 \pm 1.002^{\mathrm{b}}$ \\
\hline \% Azul & $2.73 \pm 0.140^{\mathrm{bc}}$ & $3.06 \pm 0.290^{\mathrm{c}}$ & $2.25 \pm 0.034^{\mathrm{a}}$ & $2.26 \pm 0.166^{\mathrm{ab}}$ \\
\hline
\end{tabular}

Tabla 7. Turbidez de MFT a tres niveles de presión

\begin{tabular}{|l|c|}
\hline \multicolumn{1}{|c|}{ Tratamiento } & Turbidez (NTU) \\
\hline Vino de Mora sin microfiltrar (testigo) & $63.470 \pm 1.340^{\mathrm{a}}$ \\
\hline Vino microfiltrado (1,5 bar) & $1.420 \pm 0.050^{\mathrm{b}}$ \\
\hline Vino microfiltrado (2,0 bar) & $1.240 \pm 0.020^{\mathrm{c}}$ \\
\hline Vino microfiltrado (2,5 bar) & $0.720 \pm 0.020^{\mathrm{d}}$ \\
\hline
\end{tabular}

Al aplicar bentonita sódica como pretratamiento al proceso de microfiltración, existe una evolución positiva sobre la turbidez de todas las muestras microfiltradas, observándose una reducción del 45, 43 y 39\% en comparación con el testigo, respectivamente; esto permitió considerarlas en la categoría de vino "tinto brillante" (Tabla 8). Sin embargo, el aumento del tiempo de reposo $(6,12$ y 24 horas), proporcionalmente incrementó la turbidez con diferencias significativas entre las muestras, debido a que la interacción electroestática de bentonita con los s.i.s pierde fuerza y desagrega progresivamente los complejos formados en sus compuestos originales. Un vino clarificado con bentonita puede alcanzar 9 NTU y otro por MFT de 4 a 1 NTU, lo cual proporciona al proceso dos técnicas efectivas para mejorar considerablemente la turbidez (Vilavella, 1997).

Tabla 8. Turbidez de MFT de pretratamientos con bentonita sódica

\begin{tabular}{|l|c|}
\hline Pretratamiento & Turbidez (NTU) \\
\hline SB & $1,413 \pm 0,005^{\mathrm{d}}$ \\
\hline CB-6h & $0,776 \pm 0,005^{\mathrm{a}}$ \\
\hline CB-12h & $0,803 \pm 0,005^{\mathrm{b}}$ \\
\hline CB-24h & $0,863 \pm 0,005^{\mathrm{c}}$ \\
\hline
\end{tabular}

\section{Conclusiones y recomendaciones}

La microfiltración bajo condiciones críticas de operación y de PTM, es decir, donde la colmatación es reversible y no se reducen las tasas de flujo transmembrana, hacen de esta tecnología una herramienta óptima para la clarificación de vinos; se conservan 
nutrientes, se concentran los componentes, se mantienen las características organolépticas o se mejora la estabilidad.

La adición de bentonita mejoró considerablemente el proceso sin afectar al Tono; se removieron compuestos que no podrían ser removidos con facilidad en la microfiltración y a su vez taponarían los poros de la membrana con mayor facilidad.

El tiempo de reposo óptimo de bentonita con vino como pretratamiento, deberá superar las 12 horas, para provocar solo una estabilización proteica y no afectar las características cromáticas del vino, caso contrario se están removiendo compuestos responsables de color.

La microfiltración por separado y paralelamente con un pretratamiento del vino con bentonita sódica, contribuyeron significativamente en la reducción de la turbidez, porque disminuyen considerablemente la concentración de s.i.s., a niveles bajos, mejoran el rendimiento de la MFT y alcanzan los requisitos de calidad.

Se recomienda estudiar la influencia de este tipo de tratamientos de clarificación sobre la capacidad antioxidante y el contenido de polifenoles totales.

\section{Bibliografía}

Arozarena, I. (2007). Memorias del Curso Tecnología para la elaboración de vinos de frutas. Ambato: Universidad Pública de Navarra.

Bacchin, P., Espinasse, B., \& Aimar, P. (2005). Distributions of critical flux: Modelling, experimental analysis and consequences for cross-flow membrane filtration. Journal of Membrane Science, 223-234.

Benítez, A. J. (2012). Estudio en planta piloto de la aplicación de la tecnología de membranas para el tratamiento anaerobio de Aguas Residuales Urbanas. Estudio de las Condiciones Críticas de Filtración. Valencia: Universidad Politécnica de Valencia.

Brito, B., L, P., Vera, E., \& Vaillant, F. (2010). Estudio de las Condiciones Óptimas de Operación para la Obtención de Jugo Clarificado de Granadilla ( Passiflora Ligularis L .) a través de la Microfiltración Tangencial. Quito: INIAP.

Cabrera, L., \& Perlera, A. (2009). Evaluación de la capacidad de clarificación de la arcilla de la zona de texistepeque, en vino obtenido a partir de hibiscus sabdariffa (flor de jamaica) . San Salvador: Universidad de El Salvador.

Chacón, A. V. (2005). Tecnologías de membranas en la agroindustria láctea. Agronomía Mesoamericana, 243-264.

Clarke, O. (2001). Introducción al Vino. Barcelona: NATURART, S.A.

Coronel, M. A. (2010). Estandarización y Optimización de Procesos de Vino de Mora de Castilla (Rubus glaucus Benth). Quito: Universidad Tecnológica Equinoccial.

González, I. A. (2010). Caracterización Química del Color de Diferentes Variedades de Guayaba (Psidium Guajava L.) Colombiana. Bogotá: Universidad Nacional de Colombia.

Gómez, E., Gil, R., López, J., M. De La Hera, M., \& Martínez, A. (2000). Effect of the Addition of Bentonite and Polyvinylpolypyrrolidone on the Colour and Long-term Stability of Red Wines. Journal of Wine Research, 11(3), 223-231.

Laverde, J. L. (2010). Estudio de las Condiciones Óptimas para la obtención de Jugo Clarificado de Arazá (Eugenia Stipitata), Mediante Procesos Enzimático y Membranario. Quito: Escuela Politécnica Nacional. 
Maldonado, F. (2005). Optimización del Proceso de Teñido de Telas. Quilmes: Universidad Nacional de Quilmes.

Mendoza, J. H. (2010). Uso de la Tecnología de Membranas en la Clarificación de Vinos. Cali, Colombia: Universidad del Valle.

Molina, R. (2000). Teoría de la clarificación de mostos y vinos y sus aplicaciones prácticas. Madrid: AMV- Mundi Prensa.

Montero, M. (2008). Estudio del proceso para la elaboración de jugo clarificado de mora por microfiltración tangencial. San José: Universidad de Costa Rica.

Olivero, R. E. (2006). Optimización del Proceso de Clarificación en la Elaboración de Vino de Naranja Criolla (Citrus sinensis). Mayagüez: Universidad de Puerto Rico.

Pérez, S. M., \& San José, L. G. (2006). Relationships among colorimetric parameters of rosé wines evaluated by different methods. Journal of Wine Research, 17(1), 35-43.

Peri, C., Riva, M., \& Decio, P. (1988). Crossflow Membrane Filtration of Wines: Comparison of Performance of Ultrafiltration, Microfiltration, and Intermediate Cut-Off Membranes. American Journal of Enology and Viticulture, 39(2), 162-168.

Raventós, M. (2005). Industria alimentaria. Tecnologías emergentes. Barcelona: Ediciones UPC.

Ribéreau-Gayon, P., Glories, Y., Maujean, A., \& Dubourdieu, D. (2006). Handbook of Enology, The Chemistry of Wine: Stabilization and Treatments: Second Edition. WILEY.

Rodriguez, P. (2011). Composición química y perfil sensorial de vinos de crianza de la D.O. Jumilla. Murcia: Universidad de Murcia.

Vilavella, M. (1997). La filtración tangencial en el tratamiento de vinos. Lleida: Escola Técnica Superior D'enginyeria Agraria Universitat de Lleida.

Yacelga, N. P. (2007). Obtención de jugo clarificado de mora (rubus glaucus benth) mediante Microfiltración tangencial. Quito: Escuela Politécnica Nacional. 